\title{
Expression of vascular remodelling markers in relation to bradykinin receptors in asthma and COPD
}

\author{
Fabio L M Ricciardolo, ${ }^{1}$ Federica Sabatini, ${ }^{2}$ Valentina Sorbello, ${ }^{1}$ Sabrina Benedetto, ${ }^{1}$ \\ Ilaria Defilippi, ${ }^{1}$ Loredana Petecchia, ${ }^{3}$ Cesare Usai, ${ }^{3}$ Isabella Gnemmi, ${ }^{4}$ Bruno Balbi, ${ }^{4}$ \\ Virginia De Rose, ${ }^{1}$ Nick H T ten Hacken, ${ }^{5,6}$ Dirkje S Postma, ${ }^{5,6}$ Wim Timens, \\ Antonino Di Stefano ${ }^{4}$
}

- Additional material is published online only. To view please visit the journal online (http://dx.doi.org/10.1136/ thoraxjn-2012-202741)

${ }^{1}$ Division of Respiratory Disease, Department of Clinical and Biological Sciences, A.O.U. San Luigi Hospital, University of Torino, Orbassano, Torino, Italy

${ }^{2}$ Clinical Pathology

Laboratories, Department of Experimental and Laboratory

Medicine, G. Gaslini Institute, Genova, Italy

${ }^{3}$ Institute of Biophysics,

National Research Council of Italy (CNR), Genova, Italy

${ }^{4}$ Division of Pneumology and Laboratory of

Cytoimmunopathology,

S. Maugeri Foundation, IRCCS,

Veruno, Novara, Italy

${ }^{5}$ Department of Pulmonary

Medicine and Tuberculosis,

University of Groningen,

University Medical Center

Groningen, Groningen,

The Netherlands

${ }^{6}$ GRIAC Research Institute, University of Groningen,

University Medical Center Groningen, Groningen

The Netherlands

${ }^{7}$ Department of Pathology, University Medical Center Groningen, Groningen, The Netherlands

\section{Correspondence to} Dr Fabio L M Ricciardolo, Division of Respiratory Disease, Department of Clinical and Biological Sciences, A.O.U. San Luigi Hospital, University of Torino, Orbassano, Torino 10043, Italy: fabioluigimassimo.ricciardolo@ unito.it

Received 14 September 2012 Revised 22 April 2013 Accepted 2 May 2013 Published Online First 5 June 2013

To cite: Ricciardolo FLM Sabatini F, Sorbello $V$, et al. Thorax 2013;68:803-811.

\section{ABSTRACT}

Background Vascular remodelling plays a central role in asthma and chronic obstructive pulmonary disease (COPD). Bradykinin (BK) is a vasoactive proinflammatory peptide mediating acute responses in asthma. We investigated the role of angiogenic factors in relation to $B K$ receptors in asthma and COPD.

Methods Bronchial biopsies from 33 patients with COPD, 24 old ( $\geq 50$ years) patients with ( $\geq 50$ years) asthma, 18 old control smokers, 11 old control nonsmokers, 15 young ( $\leq 40$ yrs) patients with ( $\leq 40$ years) asthma and 10 young control non-smokers were immunostained for CD31, vascular endothelial growth factor-A (VEGF-A), angiogenin and BK receptors (B2R and B1R). Fibroblast and endothelial co-localisation of relevant molecules were performed by immunofluorescence. BK-induced VEGF-A and angiogenin release was studied (ELISA) in bronchial fibroblasts from subjects with asthma and COPD.

Results In bronchial lamina propria of old patients with asthma, CD31 and VEGF-A ${ }^{+}$cell numbers were higher than old control non-smokers $(p<0.05)$. Angiogenin ${ }^{+}$, $\mathrm{B}^{2} \mathrm{R}^{+}$and $\mathrm{B} 1 \mathrm{R}^{+}$cell numbers in old patients with asthma were higher than in old control non-smokers, control smokers and patients with COPD $(p<0.01)$. Angiogenin ${ }^{+}$cell numbers were higher in patients with COPD than both old control groups $(p<0.05)$. In all patients with asthma the number of $B 2 R^{+}$cells was positively related to the numbers of $B 1 R^{+}\left(r_{s}=0.43\right)$, angiogenin ${ }^{+}\left(r_{s}=0.42\right)$ and CD31 cells $\left(r_{s}=0.46\right)$ $(p<0.01)$. Angiogenin ${ }^{+}$cell numbers were negatively related to forced expiratory volume in $1 \mathrm{~s}\left(r_{s}=-0.415\right.$, $\mathrm{p}=0.008$ ). Double immunofluorescence revealed that CD31 cells of capillary vessels coexpressed B2R and that fibroblasts coexpressed B2R, VEGF-A and angiogenin. $\mathrm{BK}\left(10^{-6} \mathrm{M}\right)$ induced significant angiogenin release in fibroblasts from asthma and to a lesser extent in COPD. Conclusions Unlike COPD, this study suggests the involvement of BK receptors in bronchial vascular remodelling in asthma.

\section{INTRODUCTION}

Asthma and chronic obstructive pulmonary disease (COPD) are chronic inflammatory and obstructive respiratory diseases associated with structural alterations in the airways. ${ }^{1}$ Angiogenesis has been recognised as a fundamental event in the development of airway remodelling that leads to irreversible airway obstruction. ${ }^{2} 3$

\section{Key messages}

\section{What is the key question?}

- Are bradykinin receptors in relation to vascular remodelling in asthma and chronic obstructive pulmonary disease?

\section{What is the bottom line?}

- Bradykinin receptors are overexpressed in the bronchial wall of old patients with asthma in conjunction with an increased expression and fibroblast-derived release of vascular growth factors.

\section{Why read on?}

- The reader will figure out the bradykinin-associated mechanisms of increased vascularity and of overexpressed vascular growth factors in old asthma.

Several proteins are potentially involved in angiogenesis, like vascular endothelial growth factor-A (VEGF-A), angiogenin and bradykinin (BK). VEGF-A is a potent multifunctional cytokine that has several effects on angiogenesis and is associated with asthma and COPD. ${ }^{2}{ }^{4}$ VEGF-A stimulates endothelial cell migration and proliferation and it is widely expressed in highly vascularised organs, including the lung. ${ }^{5}$ VEGF-A is modulated by many factors including nitric oxide and fibroblast growth factor. ${ }^{2}{ }^{6}$ Some studies have shown elevated levels of VEGF-A in bronchial biopsies, induced sputum and bronchoalveolar lavage fluid (BALF) from patients with asthma, and these correlated with increased total airway vascular area ${ }^{7-9}$ and smaller airway calibre. ${ }^{7} 9$ Furthermore, VEGF-A expression is increased in small airways of COPD. ${ }^{2}$ In addition, all VEGF isoforms showed decreased expression with age in the anterior cruciate ligament of rabbits. ${ }^{10}$

Angiogenin is a member of the ribonuclease superfamily, normally present in the blood circulation. ${ }^{11}$ It has been implicated as mitogen for vascular endothelial cells, immune modulator, activator of certain protease cascades, as well as adhesion molecule. ${ }^{11}$ Like VEGF-A, it induces vascular endothelial cell proliferation, migration and tubule formation, ${ }^{12}$ and its levels are increased in asthma. ${ }^{7}$ 
BK and the related peptide kallidin are formed from high and low molecular weight kininogen precursors following the activation of plasma and tissue kallikreins by pathophysiological stimuli leading to tissue inflammation and damage. ${ }^{14}$

The biological actions of kinins are mediated via interaction with $\mathrm{B} 2$ receptors $(\mathrm{B} 2 \mathrm{R})$ and $\mathrm{B} 1$ receptors $(\mathrm{B} 1 \mathrm{R})$. The $\mathrm{B} 2 \mathrm{R}$ is constitutively expressed on most cell types and its activation leads to various intracellular events, including nitric oxide release. ${ }^{15}$ By contrast, B1Rs can be induced during inflammatory insults. ${ }^{14} \mathrm{BK}$ induces acute inflammatory airway responses, including plasma protein extravasation/vasodilation and smooth muscle contraction, ${ }^{16}$ leading to bronchoconstriction. ${ }^{14}$ Increased levels of BK have been reported in BALF from patients with asthma, ${ }^{14}$ and it upregulates the production of VEGF-A in vitro either in human airway smooth muscle cells ${ }^{17}$ or in bronchial epithelial cells. ${ }^{18}$ Despite BK, upon stimulation of its receptors, showed a vasoactive role ${ }^{19}$ and vascular growth effect, ${ }^{18}$ no reports have been published describing possible associations between $\mathrm{BK}$ and angiogenesis in asthma and COPD. No data are available for BK B1 and B2 receptors expression in the bronchial mucosa of patients with asthma and COPD. We investigated the expression of BK B1 and B2 receptors in bronchial biopsies of patients with asthma and COPD and their association with angiogenic factors and we examined the capability of BK to release VEGF-A or angiogenin from asthmatic and COPD bronchial fibroblasts.

\section{METHODS}

\section{Study design}

This cross-sectional study has been primarily designed in order to compare COPD ( $\geq 50$ years old), asthma and control groups comparable for age. Since an age-dependent expression of VEGF isoforms and receptors in rabbits has been demonstrated, ${ }^{10}$ we decided to also include in the present study young patients with asthma (YA) and young controls ( $\leq 40$ years old) in order to evaluate potential differences related to age.

\section{Subjects}

We examined bronchial biopsies from 111 subjects by immunohistochemistry (IHC) and confocal analysis: 24 non-smoking (2 ex-smokers) old patients with asthma (OA; age $\geq 50$ years); 15 non-smoking young patients with asthma (YA; age $\leq 40$ years); 33 smoking (9 ex-smokers) patients with COPD (age $\geq 50$ years), 18 old control smokers (2 ex-smokers) (OCS; age $\geq 50$ years), 11 old control non-smokers (OCNS; age $\geq 50$ years) and 10 young control non-smokers (YCNS; age $\leq 40$ years) (table 1). Patients with asthma and the severity of asthma were identified and treated according to the Global Initiative for Asthma and American Thoracic Society criteria. ${ }^{20} 21$ Patients with COPD were staged and treated using Global Initiative for Chronic Obstructive Lung Diseases criteria. ${ }^{22}$ All control subjects had no history of respiratory disease and no airflow limitation. The clinical and demographic characteristics of all the subjects are shown in table 1 . All subjects were in stable condition (see also online supplementary material). The study conformed to the Declaration of Helsinki, was approved by the local ethics committees (A.O.U. San Luigi Hospital: n. of protocol 1759, 22 January 2008; S. Maugeri Foundation: n. of protocol p81, 20 May 2009), bronchial biopsies were performed according to the local Ethics Committee Guidelines and written informed consent was obtained from each subject.

Measurements of lung function, fibre-optic bronchoscopy, IHC, immunofluorescence and confocal microscopy, scoring system for IHC, primary cultures of bronchial fibroblasts, and ELISA tests for in vitro angiogenic factor release are described in online supplementary material.

\section{Data analysis}

Group data were expressed as mean \pm SD for lung functional data or median (range) and IQR for morphological data. We assumed a normal distribution for functional data (ie, forced expiratory volume in $1 \mathrm{~s}\left(\mathrm{FEV}_{1}\right)$, forced vital capacity, age, etc) and a non-normal distribution for morphological parameters.

Table 1 Subjects' characteristics

\begin{tabular}{|c|c|c|c|c|c|c|}
\hline \multicolumn{7}{|l|}{ Patients } \\
\hline Characteristics & Young control & Old control & Control smokers & COPD & Old asthma & Young asthma \\
\hline \multicolumn{7}{|l|}{ Subjects, $\mathrm{N}^{\circ}$} \\
\hline Total & 10 & 11 & 18 & 33 & 24 & 15 \\
\hline Male & 7 & 4 & 15 & 27 & 10 & 5 \\
\hline Female & 3 & 7 & 3 & 6 & 14 & 10 \\
\hline Age, years & $30 \pm 7$ & $69 \pm 7$ & $61 \pm 7$ & $66 \pm 8$ & $62 \pm 8$ & $26 \pm 5$ \\
\hline \multicolumn{7}{|l|}{ Smoking history } \\
\hline No & 10 & 11 & 0 & 0 & 22 & 15 \\
\hline Ex & 0 & 0 & 2 & 9 & 2 & 0 \\
\hline Current & 0 & 0 & 16 & 24 & 0 & 0 \\
\hline \multicolumn{7}{|l|}{ Atopy } \\
\hline Yes & 0 & 0 & 0 & 0 & 16 & 14 \\
\hline No & 10 & 11 & 18 & 33 & 8 & 1 \\
\hline $\mathrm{FEV}_{1}$ pre, $\%$ predicted & $104 \pm 16$ & $118 \pm 12$ & $104 \pm 13$ & $49 \pm 19^{*}$ & $74 \pm 21$ ** & $96 \pm 17^{* * *}$ \\
\hline $\mathrm{FEV}_{1}$ post, $\%$ predicted & ND & ND & ND & $55 \pm 20$ & $83 \pm 22$ & $105 \pm 12$ \\
\hline $\mathrm{FEV}_{1} / \mathrm{FVC}$ ratio, $\%$ & $82 \pm 8$ & $85 \pm 10$ & $80 \pm 6$ & $51 \pm 12^{*}$ & $74 \pm 14 * * * *$ & $83 \pm 8^{* * * *}$ \\
\hline
\end{tabular}


For this reason we applied the analysis of variance in comparing subgroups of patients and control subjects for functional data. The non-parametric Kruskal-Wallis test was applied for multiple comparisons when morphological data were analysed followed by the Mann-Whitney U test for comparison between groups. The correlation coefficient between functional-morphological data was calculated using the Spearman rank method. Data from in vitro experiments are expressed as mean \pm SEM of three independent experiments (each experiment was performed in duplicate) for each fibroblast culture. Differences between two groups were compared by using unpaired t tests. Probability values of $\mathrm{p}<0.05$ were considered statistically significant. Data analysis was performed by using the Graph Pad Prism program (GraphPad V.5.00 Software Inc, San Diego, California, USA).

\section{RESULTS}

\section{Clinical findings}

Subjects' characteristics are shown in table 1. See also online supplementary material.

\section{Inflammatory cells}

Quantification of inflammatory cells in bronchial biopsies is summarised in table 2. Data are reported in online supplementary material. IHC of all the inflammatory cells from $\mathrm{OA}$ is shown in online supplementary figure E1.

\section{Immunoreactivity of VEGF-A, CD31 and angiogenin}

VEGF-A was mainly present in fibroblasts of lamina propria and in epithelial cells (figure 1A). Cells expressing VEGF-A immunoreactivity were significantly higher in lamina propria of OA than OCNS $(p=0.0267)$ but not higher than other groups (figure 1B). VEGF-A expression was significantly higher in YA than in YCNS $(p=0.0171)$ (figure 1B). In OA $(p=0.0103)$, and also in patients with asthma as a whole $(p=0.0269)$, non-atopic subjects showed increased expression of VEGF-A in lamina propria compared with atopic subjects. Furthermore, COPD as well as OCS had higher numbers of VEGF-A ${ }^{+}$cells compared with OCNS without reaching statistical significance (figure 1B). CD31 was present in endothelial cells of lamina propria (figure 1C). The number of CD31 cells was significantly increased in OA compared with OCNS $(p=0.0378)$ and OCS $(p=0.0414)$ but it did not significantly differ with COPD (figure 1D). A significant increase of CD31 cells was observed in YCNS compared with OCNS $(p=0.0279)$.

Immunostaining for angiogenin was localised in endothelial cells and fibroblasts of lamina propria and, to a lesser extent, in epithelial cells (figure 2A). The number of angiogenin ${ }^{+}$cells was significantly higher in OA compared with OCNS $(p=0.0002)$, OCS $(p<0.0001)$ and COPD $(p<0.0001)$ (figure 2B). Similarly, the number of angiogenin ${ }^{+}$cells were also significantly elevated in patients with COPD compared with OCNS $(p=0.0090)$ and OCS $(p=0.0451)$ (figure 2B). Furthermore, angiogenin expression in lamina propria of $\mathrm{OA}$ was significantly higher than in YA $(p=0.0003)$ (figure $2 B$ ). Finally, angiogenin ${ }^{+}$cells were significantly lower in lamina propria of OCNS compared with YCNS $(\mathrm{p}=0.0496)$ (table 2). Data are reported in online supplementary material.

\section{Immunoreactivity of BK B2R and B1R}

$\mathrm{B} 2 \mathrm{R}$ was mainly present in fibroblasts within the lamina propria, in epithelial cells and in endothelial cells of capillary vessels (figure 3A). Cells expressing B2R immunoreactivity were significantly higher in lamina propria of OA compared with OCNS $(\mathrm{p}=0.0004)$, OCS $(\mathrm{p}<0.0001)$ and COPD $(\mathrm{p}<0.0001)$ (figure $3 \mathrm{~B}$ ). Similarly, immunostaining for B1R was localised in fibroblasts within the lamina propria and in endothelial cells and epithelial cells (figure 3C). Quantitative analysis showed significantly greater B1R expression in OA compared with OCNS $(p=0.0014)$, OCS $(p<0.0001)$ and COPD $(p<0.0001)$ (figure 3D), as well as in comparison with YA $(p=0.0160)$ (figure 3D). No significant differences were observed in the numbers of cells expressing B2R or B1R between any other groups studied (figure 3B,D). Data are reported in online supplementary material.

\section{Inflammatory cells, angiogenic factors, B2R and B1R expression in severe versus mild asthma}

We also investigated the differences in inflammatory cells, angiogenic markers and $\mathrm{BK}$ receptors expression in the groups of severe $(n=16)$ and mild $(n=23)$ asthma (irrespective of age). Data are reported in online supplementary material (see also online supplementary figure E2A).

Angiogenin $^{+}$cells (see online supplementary figure E2B) were significantly increased in the patients with severe asthma compared with the patients with mild asthma $(\mathrm{p}=0.019)$, whereas

Table 2 Quantification of inflammatory cells in the lamina propria of patients with COPD, asthma, control smokers and control non-smoking subjects

\begin{tabular}{|c|c|c|c|c|c|c|c|}
\hline \multicolumn{8}{|l|}{ Patients } \\
\hline & Young control & Old control & Control smokers & COPD & Old asthma & Young asthma & p Value (Kruskal-Wallis) \\
\hline CD4 & 104 (34-194) & $133(63-175)$ & $105(68-175)$ & 119 (86-139) & $180(120-296)^{*}$ & $189(86-298)$ & 0.0371 \\
\hline CD8 & $91(64-193)$ & $111(82-168)$ & $129(96-214)$ & $196(146-267)^{* *}$ & $116(83-155)$ & $52(20-111)$ & 0.0010 \\
\hline CD68 & $258(177-271)$ & $235(128-370)$ & $299(215-520)$ & $480(273-673)^{* * *}$ & $226(151-303)$ & $264(214-375)$ & 0.0016 \\
\hline Mast cells & $50(47-64)$ & $52(32-63)$ & $38(21-73)$ & $75(42-127)$ & 38 (28-97) & $94(69-132)$ & 0.1276 \\
\hline Neutrophils & $124(90-140)$ & $88(62-133)$ & $113(84-146)$ & $151(104-290)^{* * * *}$ & $241(147-309)^{* * * *}$ & $195(86-247)$ & 0.0006 \\
\hline Eosinophils & $8(0-21)$ & $15(0-26)$ & $4(0-21)$ & $6(0-35)$ & $39(35-60)^{*}$ & $32(10-115)^{*}$ & 0.0002 \\
\hline
\end{tabular}

Data are presented as median (lower quartile, LQ-upper quartile, UQ).

The Kruskal-Wallis test was applied for multiple comparisons. For comparison between groups the Mann-Whitney $U$ test was applied.

The exact $p$ values for comparison between groups are given in the Results section.

${ }^{*} p<0.05$ significantly different from young/old controls, control smokers and patients with COPD.

${ }^{* *} p<0.05$ significantly different from old controls and young/old patients with asthma.

${ }_{* * *} p<0.05$ significantly different from young/old controls, control smokers and young/old patients with asthma.

$* * * * \mathrm{p}<0.05$ significantly different from young/old controls and control smokers.

COPD, chronic obstructive pulmonary disease. 
no difference was found in the number of VEGF-A ${ }^{+}$cells, CD31 cells, B2R ${ }^{+}$cells and B1R ${ }^{+}$cells (data not shown).

\section{Correlations between angiogenic markers, BK receptors,} inflammatory cells and clinical parameters

In the whole asthma group, the number of angiogenin ${ }^{+}$cells was negatively related to $\mathrm{FEV}_{1} \quad\left(\mathrm{r}_{\mathrm{s}}=-0.415, \mathrm{p}=0.008\right.$, figure $4 \mathrm{~A}$ ) and positively related to the number of $\mathrm{B}^{2} \mathrm{R}^{+}$cells in lamina propria $\left(r_{s}=0.417, p=0.008\right.$, figure $\left.4 \mathrm{~B}\right)$. Moreover, the number of $\mathrm{B} 2 \mathrm{R}^{+}$cells in lamina propria was significantly related to the number of B1R immunoreactive cells $\left(r_{s}=0.432\right.$, $p=0.0060$, figure $4 C$ ) as well as the number of CD31 cells $\left(r_{s}=0.465, p=0.004\right.$, figure 4D). In all patients with asthma, the number of neutrophils was positively related to CD31 cells $\left(r_{s}=0.47, p=0.02\right)$, to angiogenin ${ }^{+}$cells $\left(r_{s}=0.34, p=0.04\right)$ and to $\mathrm{B}_{2} \mathrm{R}^{+}$cells in the lamina propria $\left(\mathrm{r}_{\mathrm{s}}=0.44, \mathrm{p}=0.007\right)$.

No other statistically significant correlations were found between angiogenic markers, BK receptors, inflammatory cells or any clinical parameters.

\section{Immunofluorescence with confocal microscopy double} staining for localisation of BK B2 receptor in CD31 cells

Double immunofluorescence staining of B2R with CD31 was performed using laser-scanning confocal microscopy. Endothelial cells of capillary vessels in lamina propria of OA were immunoreactive for CD31 (figure 5A). Epithelial cells and endothelial cells of capillary vessels in lamina propria were immunoreactive for B2R (figure $5 \mathrm{~B}$ ). Merging of the red (B2R) and green (CD31) images revealed that CD31 cells of capillary vessels coexpressed $\mathrm{B} 2 \mathrm{R}$ (figure $5 \mathrm{C}$ ).

\section{Immunofluorescence with confocal microscopy double staining for identification of 5B5 cells coexpressing BK B2 receptor and angiogenic growth factors}

Double immunofluorescence staining of B2R, VEGF-A or angiogenin with 5B5 (marker for fibroblast) was performed using laser-scanning confocal microscopy. The large majority of cells in lamina propria of $\mathrm{OA}$ was immunoreactive for 5B5 (figure 6A,E,I). Merging of the red (B2R, VEGF-A or angiogenin) and green (5B5) images revealed that $5 \mathrm{~B}^{+}$cells in lamina propria coexpressed B2R (C), VEGF-A (G) or angiogenin $(\mathrm{M})$. Co-localisation quantitative analysis (figure $6 \mathrm{D}, \mathrm{H}, \mathrm{N}$ ) confirmed 5B5 and B2R $(72 \pm 12 \%)$ coexpression as well as 5B5 and VEGF-A $(87 \pm 2 \%)$ or angiogenin $(80.5 \pm 3.5)$ co-localisation in lamina propria of OA.

BK-induced VEGF-A and angiogenin release by HBF in vitro Unstimulated human bronchial fibroblasts (HBF) from OA $(n=3)$ and COPD $(n=3)$ release VEGF-A and angiogenin (figure 7). Incubation with $\mathrm{BK}\left(10^{-6} \mathrm{M}\right)$ induced a significant
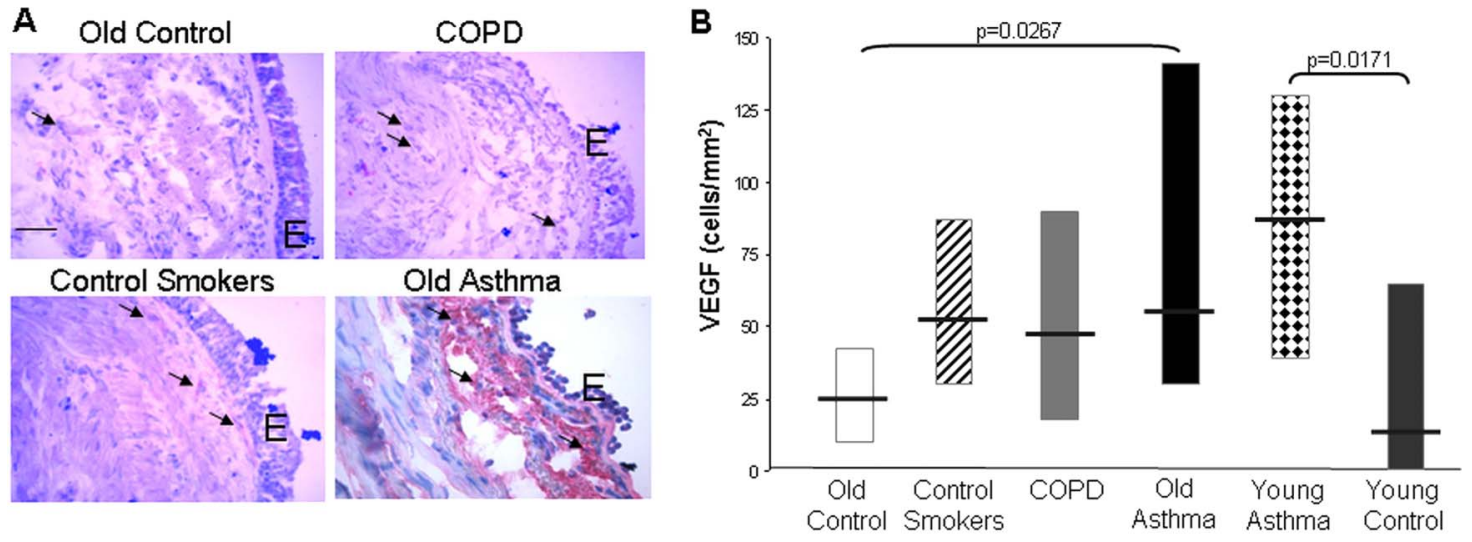



Control Smokers



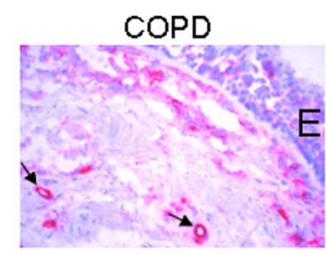

Old Asthma

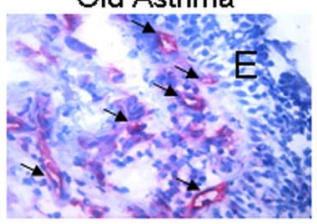

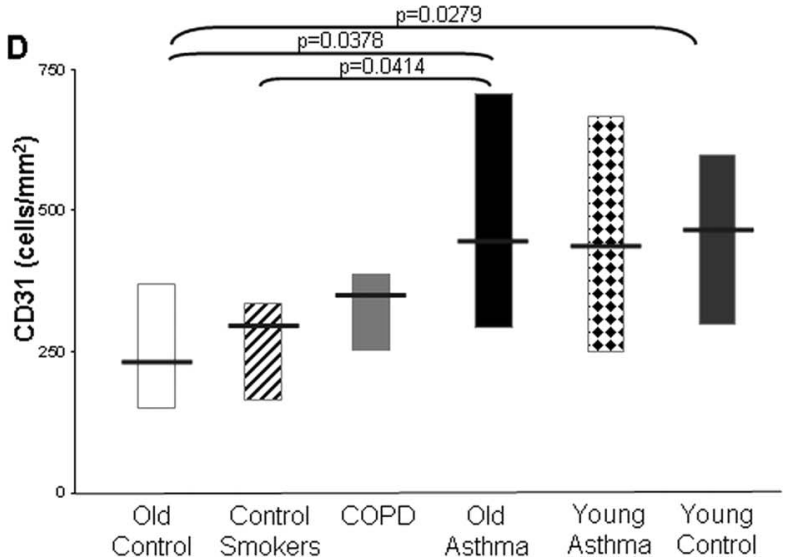

Figure 1 Vascular endothelial growth factor-A (VEGF-A) (A and B) and CD31 (C and D) expression in bronchial biopsy specimens obtained from old control non-smokers, healthy smokers, patients with chronic obstructive pulmonary disease (COPD), old and young patients with asthma, young control non-smokers. (A and C) Photomicrographs showing the bronchial immunostaining for VEGF-A and CD31, respectively, are representative of those from 11 old control non-smokers, 18 healthy smokers, 33 patients with COPD and 24 old patients with asthma. Original magnification 400x. Internal scale: $25 \mu \mathrm{m}$. E, epithelium. (B and D) Number of VEGF-A ${ }^{+}$and CD31 cells/mm $/ \mathrm{mm}^{2}$ of bronchial lamina propria from old control non-smokers, healthy smokers, patients with COPD, old and young patients with asthma, young control non-smokers. Each bar indicates the median value in the respective groups and boxes represent lower and upper quartiles. The comparisons are made on the basis of age (old groups or young groups) and between asthma or control groups. Access the article online to view this figure in colour. 

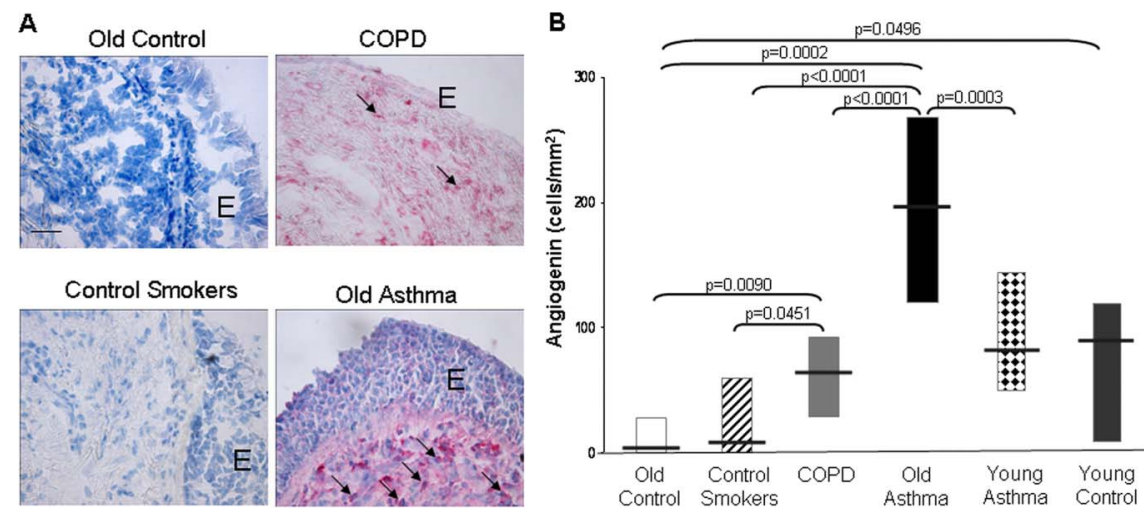

Figure 2 Angiogenin expression in bronchial biopsy specimens obtained from old control non-smokers, healthy smokers, patients with chronic obstructive pulmonary disease (COPD), old and young patients with asthma, young control non-smokers. (A) Photomicrographs showing the bronchial immunostaining for angiogenin are representative of those from 11 old control non-smokers, 18 healthy smokers, 33 patients with COPD and 24 old patients with asthma. Original magnification 400x. Internal scale: $25 \mu \mathrm{m}$. E, epithelium. (B) Number of angiogenin ${ }^{+}$cells $/ \mathrm{mm}^{2}$ of bronchial lamina propria from old control non-smokers, healthy smokers, patients with COPD, old and young patients with asthma, young control non-smokers. Each bar indicates the median value in the respective groups and boxes represent lower and upper quartiles. The comparisons are made on the basis of age (old groups or young groups) and between asthma or control groups. Access the article online to view this figure in colour.
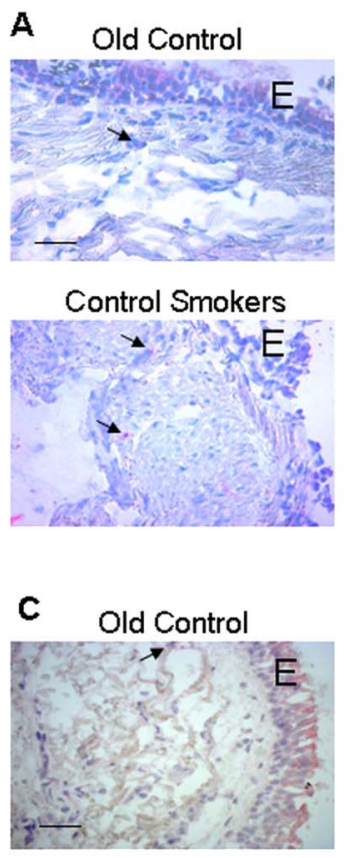

Control Smokers

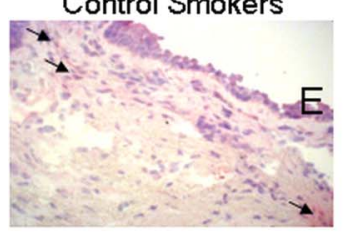

COPD

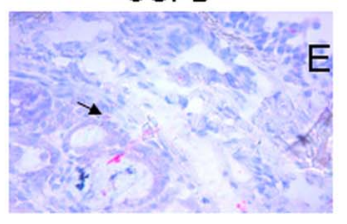

Old Asthma

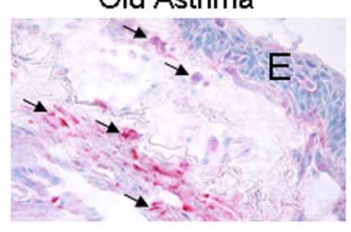

COPD

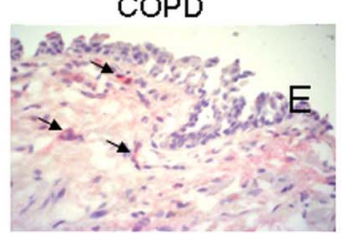

Old Asthma

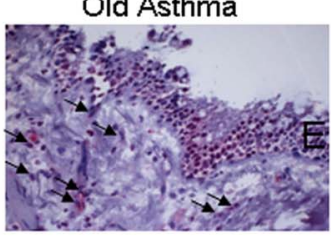

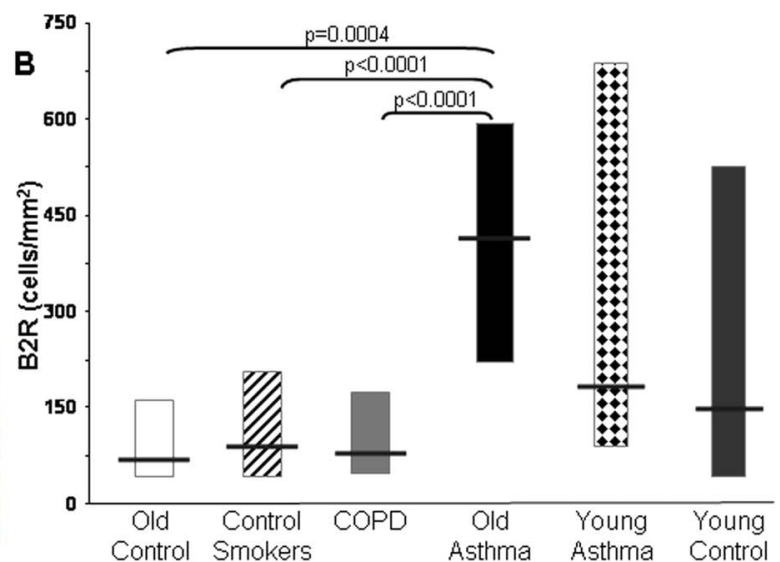

$\mathrm{p}=0.0014$

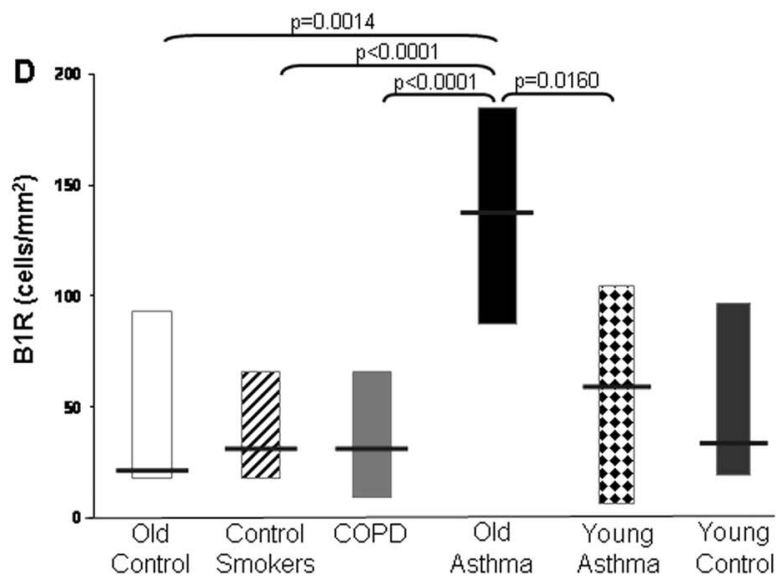

Figure 3 Bradykinin B2 receptor (B2R) (A and B) and bradykinin B1 receptor (B1R) (C and D) expression in bronchial biopsy specimens obtained from old control non-smokers, healthy smokers, patients with chronic obstructive pulmonary disease (COPD), old and young patients with asthma, young control non-smokers. ( $A$ and $C$ ) Photomicrographs showing the bronchial immunostaining for B2R and B1R, respectively, are representative of those from 11 old non-smokers, 18 healthy smokers, 33 patients with COPD and 24 old patients with asthma. Original magnification 400x. Internal scale: $25 \mu \mathrm{m}$. $E=$ =epithelium. ( $B$ and D) Number of $B 2 R^{+}$and $B 1 R^{+}$cells $/ \mathrm{mm}^{2}$ of bronchial lamina propria from old control non-smokers, healthy smokers, patients with COPD, old and young patients with asthma, young control non-smokers. Each bar indicates the median value in the respective groups and boxes represent lower and upper quartiles. The comparisons are made on the basis of age (old groups or young groups) and between asthma or control groups. Access the article online to view this figure in colour. 

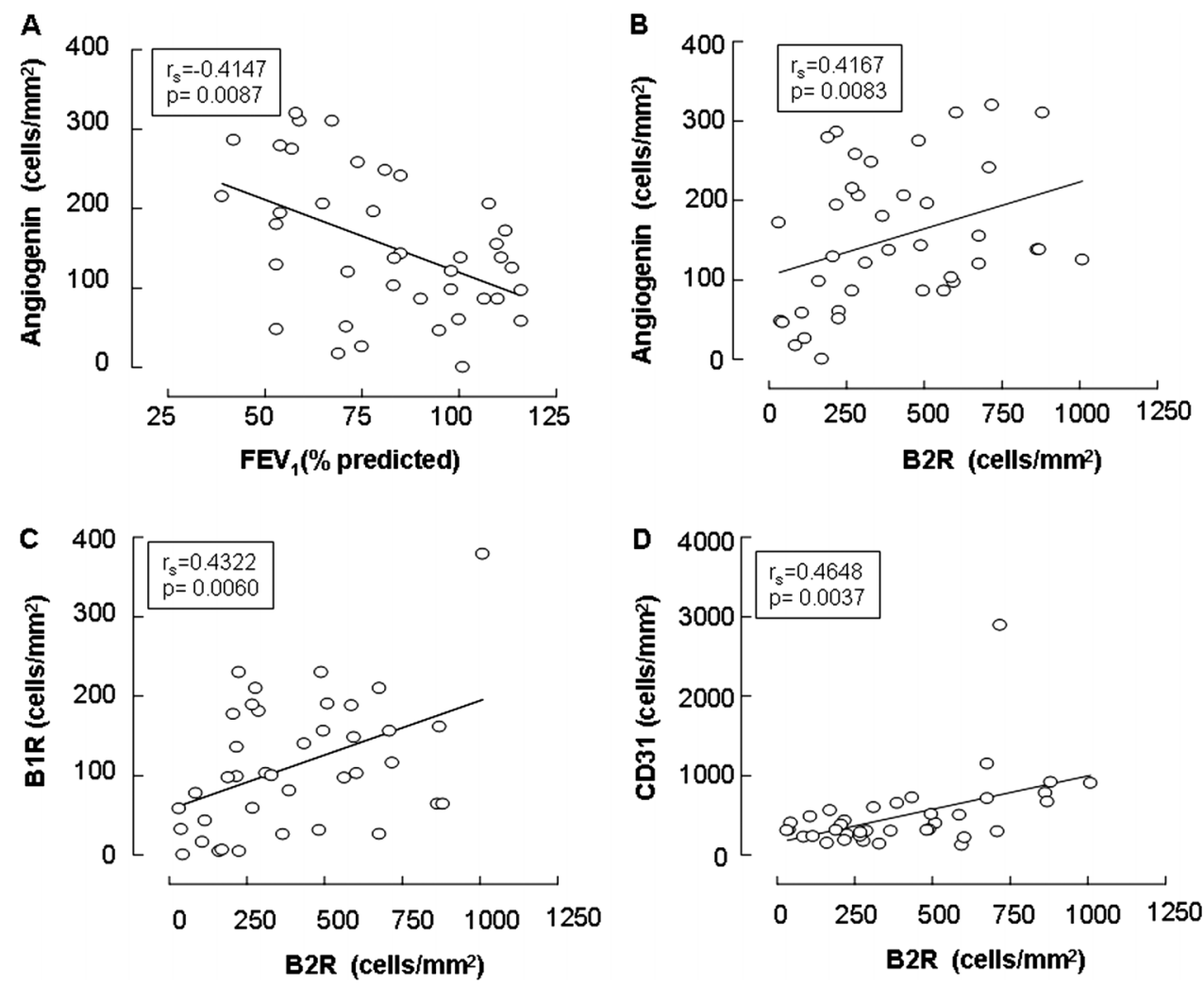

Figure 4 Relationship between angiogenin positive cells and forced expiratory volume in $1 \mathrm{~S}\left(F E V_{1}\right)\left(\%\right.$ predicted) levels $(A)$ or the number of $B 2 R^{+}$ cells (B) in the bronchial lamina propria of all patients with asthma. In (C-D) the correlation between $B 2 \mathrm{R}^{+}$cells number and B1R immunoreactive cells (C) or the number of CD31 cells (D) in the bronchial lamina propria of all patients with asthma are shown. The correlation coefficient was obtained using the Spearman rank method $\left(r_{s}\right)$.

increase in VEGF-A (figure 7A: $p=0.0248$; figure 7C: $p=0.016$ ) and angiogenin release (figure 7B: $p<0.0001$; figure 7D: $p<0.0001)$ in HBF from patients with asthma and COPD, respectively. BK (1 h exposure) similarly increased VEGF-A levels in HBF from patients with asthma $(160 \pm 25 \%)$ and COPD (208 $\pm 34 \%$; p $>0.05)$, while BK elevated angiogenin levels differently in asthma fibroblasts $(669 \pm 111 \%)$ compared with COPD fibroblasts $(280 \pm 10 \% ; \mathrm{p}=0.013)$. Details of these experiments are reported in online supplementary material.

\section{DISCUSSION}

This study demonstrates that BK receptors (B2R and B1R) are upregulated in the lamina propria of OA compared with agecomparable healthy control subjects and COPD in conjunction with increased number of vessels (CD31 cells) and expression of angiogenin and, to a lesser extent, VEGF-A. Furthermore, angiogenin expression was significantly increased in patients with severe compared with mild asthma. Angiogenin was also negatively related to $\mathrm{FEV}_{1}$ in all patients with asthma and positively related to B2R expression. Finally, we showed localisation of B2R, VEGF-A and angiogenin in bronchial fibroblasts from $\mathrm{OA}$ and the ability of BK to release VEGF-A and angiogenin in bronchial fibroblasts from patients with asthma and, to a lesser extent, from patients with COPD.

Asthma and COPD are chronic inflammatory diseases of the lung associated with structural remodelling that contributes to airway obstruction (see also online supplementary material). We found significant and positive correlations between numbers of neutrophils and $\mathrm{B}_{2} \mathrm{R}^{+}, \mathrm{CD} 31$ or angiogenin ${ }^{+}$cells suggesting a role for this inflammatory cell in vascular remodelling of OA.
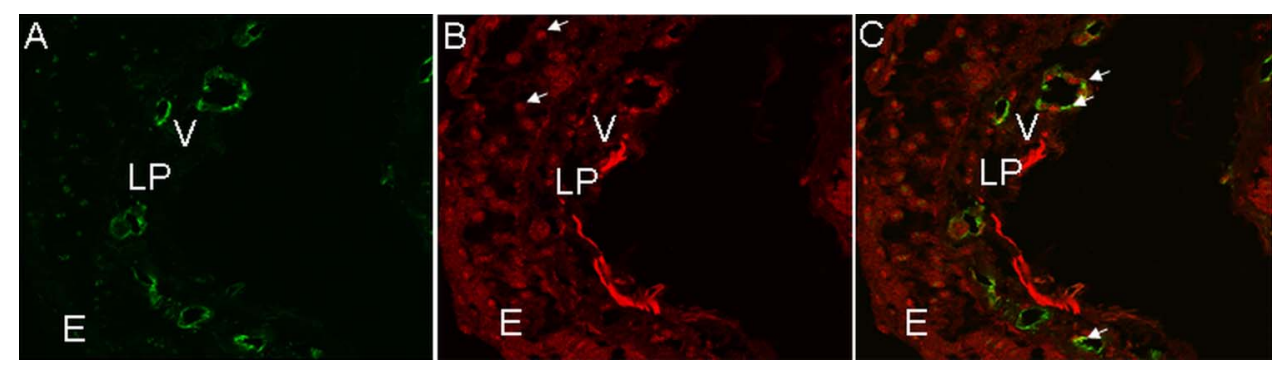

Figure 5 Representative photomicrographs obtained by confocal microscopy after immunofluorescence staining of CD31 and bradykinin B2 receptor (B2R) in bronchial lamina propria from an old patient with asthma. In (A) Alexa Fluor 488-green staining represents CD31, in (B) Alexa Fluor 647-red staining represents B2R whereas co-localised pixels are displayed in yellow (C). Arrows indicate B2R and B2R/CD31 positive cells (B and $C$, respectively). Original magnification: $630 \times$. E, epithelium; LP, lamina propria; V, vessel. Access the article online to view this figure in colour. 
Co-localisation
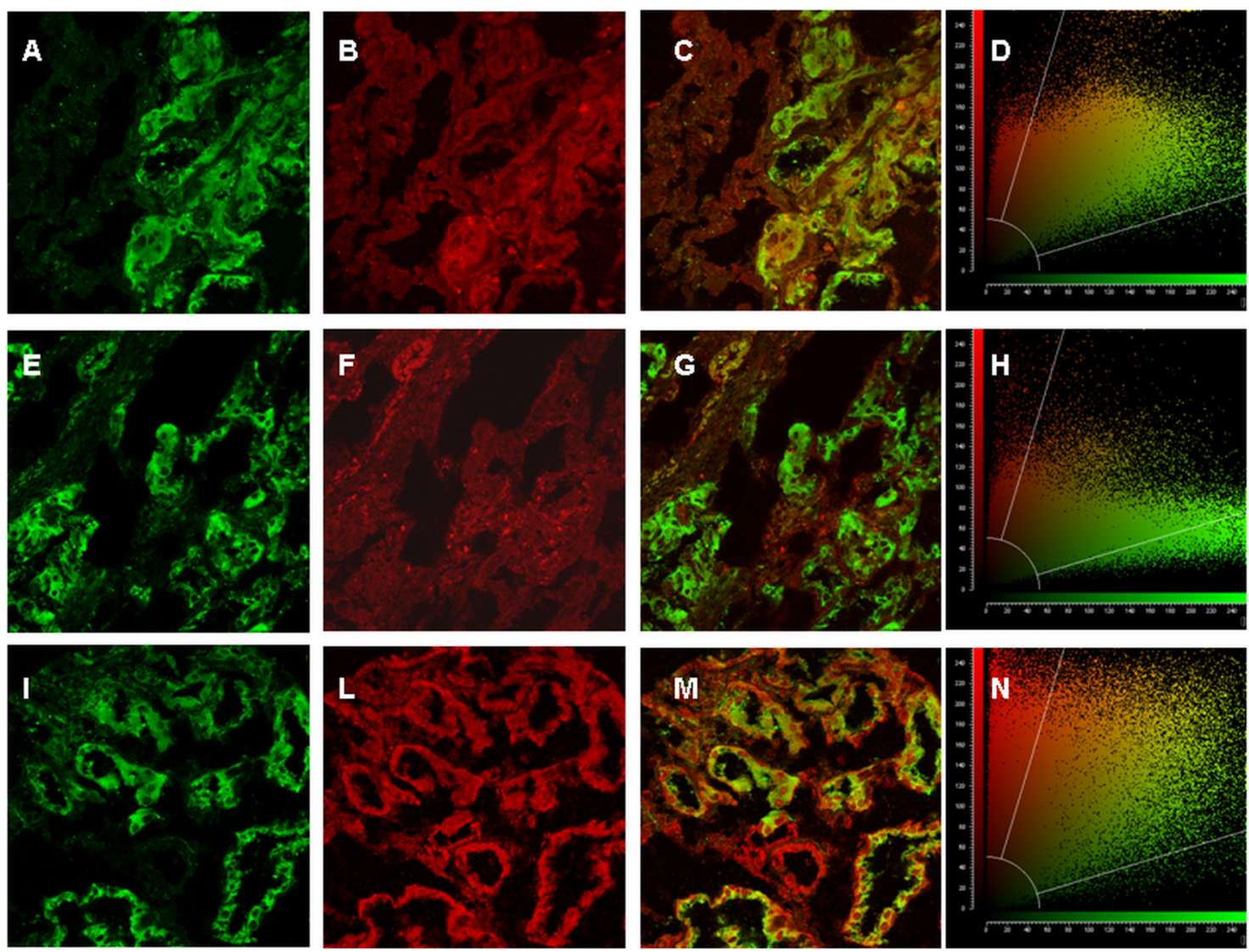

Figure 6 Representative photomicrographs obtained by confocal microscopy after immunofluorescence staining of prolyl 4-hydroxylase (5B5), bradykinin B2 receptor (B2R), vascular endothelial growth factor-A (VEGF-A) and angiogenin expression in bronchial lamina propria from an old patient with asthma. (A, E and I) show, the Alexa Fluor 488-tagged anti-5B5 (green) whereas ( $\mathrm{B}, \mathrm{F}$ and $\mathrm{L}$ ) represent Alexa Fluor 647-tagged anti-B2R, anti-VEGF-A or antiangiogenin (red) respectively. In (C, G and M) the co-localised pixels representing coexpression of 5B5 and B2R (C), VEGF-A (G) or angiogenin (M) are displayed in yellow. (, $\mathrm{H}$ and $N$ ) show the correlation cytofluorogram of the images in $(A$ and $B$, or $E$ and $F$ or I and $\mathrm{L})$, respectively. Original magnification $630 \times$. Access the article online to view this figure in colour.

Figure 7 Effect of bradykinin (BK) B2 receptor antagonist HOE140 on BK-induced vascular endothelial growth factor-A (VEGF-A) release $(A$ and $C)$ and angiogenin release ( $B$ and $D$ ) by human bronchial fibroblasts from patients with asthma $(\mathrm{n}=3 ; \mathrm{A}$ and $\mathrm{B})$ and from chronic obstructive pulmonary disease $(n=3$; C and D). Cells were pretreated with or without HOE140 $(1 \mu \mathrm{M})$ for $30 \mathrm{~min}$ before incubation with BK $\left(10^{-6} \mathrm{M}\right)$ for $1 \mathrm{~h}$. At the end of the incubation period, the culture supernatants were collected and the VEGF-A or angiogenin contents were evaluated by ELISA. Data are presented as mean \pm SEM of three independent experiments for each fibroblast culture. ${ }^{*} p<0.05,{ }^{* *} p<0.001$ versus unstimulated cells; and ${ }^{\#} p<0.05$, \#\# $p<0.01,{ }^{\# \#} p<0.001$ versus cells stimulated with BK.
A

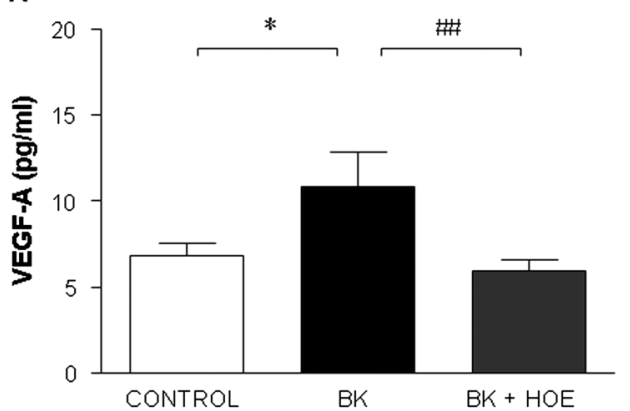

C

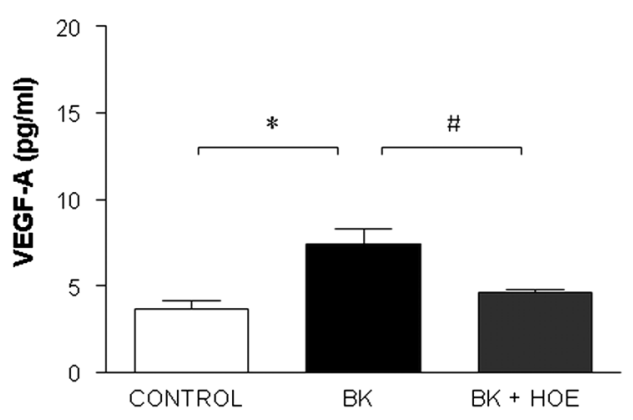

B

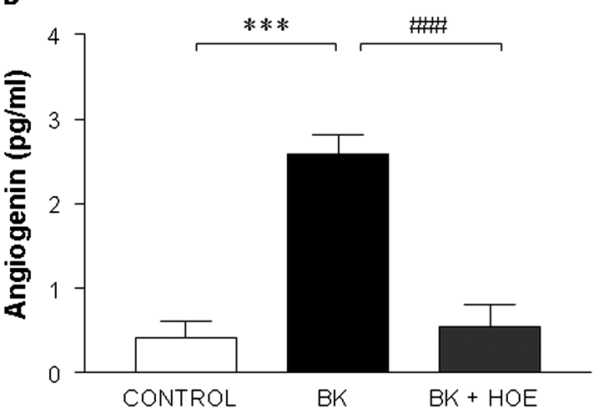

D




In a previous study BK-induced interleukin 8 production due to B2R stimulation in lung fibroblasts is in part dependent on the activation of ERK1/2 and p38 MAPK-pathway. ${ }^{23}$ These data, together with our report on positive correlations between neutrophils and angiogenic molecules suggest a role for neutrophil-released mediators (including proteases) in modulating angiogenesis in asthma through activation of protease activated receptors. $^{24}$

Vascular remodelling and reactivity of the airways including angiogenesis, vasodilation and microvascular leakage are the most prominent and uniform findings in asthma. ${ }^{2}$ Furthermore, bronchial vascular changes may also occur in COPD, particularly in the small airways. ${ }^{25}$ Microvascular changes in asthma and COPD may contribute to an increase in airway wall thickness which may be associated with severity of airway obstruction and disease progression. ${ }^{25}$ Angiogenesis is a complex multiphase process involving a great number of growth factors, cytokines, chemokines and other factors. Some bronchial biopsy studies ${ }^{78}$ as well as induced sputum studies ${ }^{9}$ observed higher VEGF-A levels, related to vascularity, in asthmatic airways than those of healthy controls. ${ }^{78}$ Angiogenin induces vascular endothelial cell proliferation, migration and tubule formation. In biopsies from patients with asthma, a higher expression of basic fibroblast growth factor and angiogenin was reported, with significant correlations between the vascular area and the number of angiogenic factor-positive cells within the airways. ${ }^{7}$ Furthermore, VEGF-A and angiogenin are able to promote in vitro angiogenesis expressed as induction of primitive vascular tubule structures in culture with BALF collected from patients with mild asthma. ${ }^{13} \mathrm{~A}$ recent study also showed increased levels of VEGF-A and angiogenin in induced sputum from stable COPD compared with healthy smokers and non-smokers. ${ }^{26}$ We extend these observations by comparing patients with asthma, COPD and controls in relation to age. This is the first study in humans showing that the number of CD31 and angiogenin ${ }^{+}$cells was significantly reduced in old compared with young controls demonstrating that vascularity is probably influenced by age per se. VEGF-A appears to be more expressed in lamina propria of OA while angiogenin is significantly higher in OA (also in comparison with YA) and in COPD. Furthermore, angiogenin ${ }^{+}$cell expression in lamina propria negatively correlated with the level of $\mathrm{FEV}_{1}$ in the group of patients with asthma, suggesting that angiogenin expression in lamina propria is associated with the severity of asthma, confirming previous findings. ${ }^{25}$ The modest increase of angiogenin in the bronchi of our COPD may be related to apoptosis of endothelial cells induced by VEGF-A reduction due to oxidative stress as described elsewhere. ${ }^{27}$ Finally, we showed for the first time a marked increase of VEGF-A expression in the lamina propria of non-atopic patients with asthma compared with atopic patients with asthma suggesting that in 'intrinsic' asthma higher release of VEGF-A could specifically contribute to the formation of vascular remodelling scenario.

BK exerts several effects in airways through direct and indirect pathways which are mediated by activation of two main BK receptor subtypes, B1 and B2, that differ in expression and ligand specificity. In tissues B1 receptors are inducible, whereas B2 receptors are constitutively expressed. ${ }^{14}$ The distribution of B2 receptors has been mapped out in human and guinea pig lung by autoradiography with $\left[{ }^{3} \mathrm{H}\right] \mathrm{BK} .{ }^{28}$ See also online supplementary material. In this study, for the first time we demonstrated the expression of $\mathrm{BK} \mathrm{B} 1 \mathrm{R}$ and $\mathrm{B} 2 \mathrm{R}$ in fibroblasts, epithelial cells and endothelial cells of the human bronchial lamina propria (by IHC and immunofluorescence analysis), and that their total expression is significantly increased in OA compared with OCNS, OCS and COPD (and for B1R also compared with YA), suggesting a potential role for these receptors in the vascular remodelling of old asthma. BK promotes angiogenesis by upregulation of basic fibroblast growth factor through the B1 receptor or by stimulation of VEGF-A formation following B2 receptor stimulation. ${ }^{17}{ }^{29}$ In our study, B2R showed a positive correlation with $\mathrm{B} 1 \mathrm{R}$ and with CD31 endothelial cells and angiogenin suggesting that $\mathrm{B} 2 \mathrm{R}$ may promote angiogenesis leading to blood vessel neoformation. We also found by immunofluorescence that the large majority of bronchial fibroblasts in OA coexpressed B2R, angiogenin and VEGF-A indicating a major role for fibroblasts in producing vascular growth factors potentially released upon stimulation of BK B2R.

$\mathrm{BK}$ is involved in the repair processes inducing fibroblast proliferation and differentiation. ${ }^{30} \mathrm{BK}$ also regulates angiogenic growth factors expression (angiogenin and VEGF-A) in human dental pulp fibroblasts. ${ }^{31}$ Other authors showed that human airway smooth muscle cells secrete VEGF-A and this secretion is induced by $\mathrm{BK}$ via $\mathrm{B} 2 \mathrm{R} .{ }^{17}$ In our in vitro study, we demonstrated for the first time that BK induced VEGF-A and angiogenin release in primary cultures of $\mathrm{HBF}$ from patients with asthma and that this effect is mediated by $\mathrm{B} 2 \mathrm{R}$, as confirmed by the use of the B2R antagonist HOE140 which completely blocked BK-induced VEGF-A and angiogenin release. We also showed similar effects, but to a lesser extent, in bronchial fibroblasts from COPD suggesting that bronchial fibroblasts from patients with asthma are more prone to release angiogenic factors.

In conclusion, this study demonstrates an increased expression of BK receptors in relation to vascular remodelling in asthma and suggests a role for bronchial asthmatic fibroblasts in releasing vascular growth factors upon activation of BK B2 receptors. Our study showed that markers of vascular remodelling are major features of $\mathrm{OA}$ and are potentially involved in fixed airway obstruction by increasing airway wall thickness and stiffness. The increased vascular reactivity of asthmatic airways, due to stimuli like $\mathrm{BK}^{32}$ in disease-related vascular remodelling might participate in airway variability which is a typical pattern of airway patency in asthma. Previous observations showed kinin involvement in airway hyperresponsiveness in asthma, ${ }^{33}$ and the capability of selective B2R antagonists to treat acute attacks of hereditary angioedema, ${ }^{34}$ and to improve pulmonary function in patients with asthma. ${ }^{35}$ These data, combined, lead us to encourage the planning of long term studies using these selective antagonists in asthma treatment in order to inhibit BK B2 receptor-mediated angiogenic effects and its related remodelling processes.

Acknowledgements The authors thank Dr Gian Mario Massaglia for his contribution in collecting bronchial biopsies.

Contributors All authors included in this paper fulfil the criteria of authorship. In addition we assure that there is no one else who fulfils the criteria that has not been included as an author.

Funding This study was supported by MIUR ex-60\% 2008.

Competing interests None.

Patient consent Obtained.

Ethics approval The study conformed to the Declaration of Helsinki. Ethics consent was obtained, bronchial biopsies were performed according to the local ethics committee guidelines.

Provenance and peer review Not commissioned; externally peer reviewed.

\section{REFERENCES}

1 Jeffery PK. Remodeling in asthma and chronic obstructive lung disease. Am J Respir Crit Care Med 2001;164:S28-38. 
2 Postma DS, Timens W. Remodeling in asthma and chronic obstructive pulmonary disease. Proc Am Thorac Soc 2006;3:434-9.

3 Hashimoto M, Tanaka $\mathrm{H}$, Abe S. Quantitative analysis of bronchial wall vascularity in the medium and small airways of patients with asthma and COPD. Chest 2005; 127:965-72.

4 Asosingh K, Erzurum SC. Angioplasticity in asthma. Biochem Soc Trans 2009;37:805-10.

5 Thebaud B, Ladha F, Michelakis ED, et al. Vascular endothelial growth factor gene therapy increases survival, promotes lung angiogenesis, and prevents alveolar damage in hyperoxia-induced lung injury: evidence that angiogenesis participates in alveolarization. Circulation 2005;112:2477-86.

6 Presta $M$, Andres $G$, Leali $D$, et al. Inflammatory cells and chemokine sustain FGF2-induced angiogenesis. Eur Cytokine Netw 2009;20:39-50.

7 Hoshino M, Takahashi M, Aoike N. Expression of vascular endothelial growth factor, basic fibroblast growth factor, and angiogenin immunoreactivity in asthmatic airways and its relationship to angiogenesis. J Allergy Clin Immunol 2001;107:295-301.

8 Feltis BN, Wignarajah D, Zheng L, et al. Increased vascular endothelial growth factor and receptors: relationship to angiogenesis in asthma. Am J Respir Crit Care Med 2006;173:1201-7.

9 Asai $\mathrm{K}$, Kanazawa $\mathrm{H}$, Kamoi $\mathrm{H}$, et al. Increased levels of vascular endothelial growth factor in induced sputum in asthmatic patients. Clin Exp Allergy 2003;33:595-9.

10 Hofstaetter JG, Saad FA, Sunk IG, et al. Age-dependent expression of VEGF isoforms and receptors in the rabbit anterior cruciate ligament. Biochim Biophys Acta 2007:1770:997-1002.

11 Adams SA, Subramanian V. The angiogenins: an emerging family of ribonuclease related proteins with diverse cellular functions. Angiogenesis 1999;3:189-99.

12 Kishimoto K, Liu S, Tsuji T, et al. Endogenous angiogenin in endothelial cells is a general requirement for cell proliferation and angiogenesis. Oncogene 2005;24:445-56.

13 Simcock DE, Kanabar V, Clarke GW, et al. Proangiogenic activity in bronchoalveolar lavage fluid from patients with asthma. Am J Respir Crit Care Med 2007;176:146-53.

14 Abraham WM, Scuri M, Farmer SG. Peptide and non-peptide bradykinin receptor antagonists: role in allergic airway disease. Eur J Pharmacol 2006;533:215-21.

15 Ricciardolo FL, Vergnani L, Wiegand S, et al. Detection of nitric oxide release induced by bradykinin in guinea pig trachea and main bronchi using a porphyrinic microsensor. Am J Respir Cell Mol Biol 2000;22:97-104.

16 Figini $\mathrm{M}$, Ricciardolo $\mathrm{FL}$, Javdan $\mathrm{P}$, et al. Evidence that epithelium-derived relaxing factor released by bradykinin in the guinea pig trachea is nitric oxide. Am J Respir Crit Care Med 1996;153:918-23.

17 Knox AJ, Corbett L, Stocks J, et al. Human airway smooth muscle cells secrete vascular endothelial growth factor: up-regulation by bradykinin via a protein kinase C and prostanoid-dependent mechanism. FASEB 2001;15:2480-8.

18 Koyama S, Sato E, Tsukadaira A, et al. Vascular endothelial growth factor mRNA and protein expression in airway epithelial cell lines in vitro. Eur Respir 2002;20:1449-56.
19 Ricciardolo FL, Nadel JA, Bertrand C, et al. Tachykinins and kinins in antigen-evoked plasma extravasation in guinea-pig nasal mucosa. Eur J Pharmacol 1994:261:127-32.

20 Global Strategy for Asthma Management and Prevention. Global Initiative for Asthma (GINA). NHLBI/WHO workshop report. Bethesda, National Heart, Lung and Blood Institute. http://www.ginasthma.org/Updated 2007.

21 Wenzel SE, Fahy JV, Irvin CG, et al. Proceedings of the ATS Workshop on Refractory Asthma: current understanding, recommendations and unanswered questions. Am J Respir Crit Care Med 2000;162:2341-51.

22 Global Initiative for Chronic Obstructive Lung Disease. Global Strategy for the Diagnosis, Management and Prevention of Chronic Obstructive Pulmonary Disease. NHLBI/WHO workshop report. ethesda, National Heart, Lung and Blood Institute. http://www.goldcopd.org/ Updated 2010

23 Hayashi R, Yamashita N, Matsui S, et al. Bradykinin stimulates IL-6 and IL-8 production by human lung fibroblasts through ERK- and p38 MAPK-dependent mechanisms. Eur Respir J 2000;16:452-8.

24 Wheeler-Jones CP, Farrar C, Garonna E. Protease-activated receptors, cyclo-oxygenases and pro-angiogenic signalling in endothelial cells. Biochem Soc Trans 2009;37:1179-83.

25 Zanini A, Chetta A, Imperatori AS, et al. The role of the bronchial microvasculature in the airway remodeling in asthma and COPD. Resp Res 2010;11:1-11.

26 Kristan SS, Marc MM, Kern I, et al. Airway angiogenesis in stable and exacerbated chronic obstructive pulmonary disease. Scand I Immunol 2011;75:109-14

27 Demedts IK, Demoor T, Bracke KR, et al. Role of apoptosis in the pathogenesis of COPD and pulmonary emphysema. Respir Res 2006;7:53.

28 Mak JCW, Barnes PJ. Autoradiographic visualization of bradykinin receptors in human and guinea pig lung. Eur J Pharmacol 1991;194:37-43.

29 Colman RW. Regulation of angiogenesis by the kallikrein-kinin system. Curr Pharm Des 2006;12:2599-607.

30 Petecchia L, Sabatini F, Usai C, et al. Mechanisms of bradykinin-induced contraction in human fetal lung fibroblast. Eur Respir J 2010;36:1-10.

31 El Karim IA, Linden GJ, Irwin CR, et al. Neuropeptides regulate expression of angiogenic growth factors in human dental pulp fibroblasts. J Endod 2009;35:829-33.

32 Chung KF, Rogers DF, Barnes PJ, et al. The role of increased airway microvascular permeability and plasma exudation in asthma. Eur Respir J 1990;3:329-37.

33 Ricciardolo FLM, Geppetti P, Mistretta A, et al. Randomised double-blind placebo-controlled study of the effect of inhibition of nitric oxide synthesis in bradykinin-induced asthma. Lancet 1996;348:374-7.

34 Bork K, Frank J, Grundt B, et al. Treatment of acute edema attacks in hereditary angioedema with a bradykinin receptor-2 antagonist (Icatibant). J Allergy Clin Immunol 2007;119:1497-503.

35 Akbarg AM, Wirth KJ, Schoelkens BA. Efficacy and tolerability of Icatibant (HOE 140 ) in patients with moderately severe chronic bronchial asthma. Immunopharmacology 1996;33:238-42. 